\title{
Scaling of the size of the first agonist EMG burst during rapid wrist movements in patients with Parkinson's disease
}

\author{
A BERARDELLI,* J P R DICK, J C ROTHWELL, B L DAY, C D MARSDEN
}

From the MRC Movement Disorders Research Group, Department of Neurology and Parkinson's Disease Society Research Centre, Institute of Psychiatry and King's College Hospital Medical School, London, UK

SUMMARY Rapid wrist flexion movements were studied in a group of 10 patients with Parkinson's disease both on and off their normal drug therapy, and were compared with the same movements made by a group of eight normal individuals. When normal subjects made movements through $60^{\circ}$, the first agonist burst of EMG activity in the wrist flexor muscles was longer and larger than that seen in movements of $15^{\circ}$. If a large opposing load of $2.2 \mathrm{Nm}$ was added, this also increased the size and duration of the first agonist EMG burst. Although the movements made by the patients were slower than those of normals, the size and duration of the first agonist EMG burst changed with movement size and added load in the normal way. This shows that patients can produce large, long bursts of EMG activity, but that there is a failure to match these parameters appropriately to the size of movement required. The effect of levodopa therapy on the movements was not dramatic. Although patients produced faster wrist movements when on medication than when off, the change was relatively small compared with the change seen in their overall clinical rating. Changes in the velocity of movements at a single joint are not a good reflection of the overall clinical state of patients with Parkinson's disease.

Patients with Parkinson's disease cannot move as rapidly as normal individuals. Physiological studies on the arm have shown that movements at both proximal ${ }^{1-3}$ and distal ${ }^{4}$ joints are affected. In normal individuals such movements are made with a single triphasic pattern of EMG activity in agonist, antagonist and agonist muscles. The first agonist burst provides the impulsive force for the movement, and the antagonist activity provides a braking force to halt the limb. In bradykinetic patients with Parkinson's disease the first agonist burst is often not large enough to move the limb through the desired angle, so additional bursts of EMG activity are required to achieve the target. Hallett and Khoshbin ${ }^{2}$ suggested

\footnotetext{
*Permanent address: Quinta Clinica Neurologica, Dipartimento di Scienze Neurologiche, Universita di Roma "La Sapienz", Viale dell' Universita 30, Roma, Italia.
}

Address for reprint requests: Professor CD Marsden, Department of Neurology, Institute of Psychiatry, De Crespigny Park, London SE5 8AF, UK.

Received 6 June 1985 and in revised form 27 September 1985. Accepted 28 September 1985 that failure to activate or appropriately "energise" the agonist muscle was responsible for the bradykinesia of Parkinson's disease.

The reason for the reduction in size of the first agonist burst in Parkinson's disease is not clear. One possibility is that it is due to saturation in the mechanism that produces the burst. If this were true then patients with Parkinson's disease would be unable to produce large bursts of agonist activity under any circumstances. Up to a certain size, small movements might be performed relatively normally, ${ }^{3}$ but large movements above the level of agonist saturation would be slow and require extra cycles of EMG activity. Failure to "energise" muscles would only be apparent in large movements.

We have tested this hypothesis by examining the behaviour of the first agonist burst for rapid wrist movements under a range of conditions which normally produce changes in its size and duration. ${ }^{5}$ We find that although the velocity of all movements is slow in Parkinsonian patients, the size of the first agonist burst can be modulated in the normal way. We conclude that agonsit burst size does not saturate, but that it is inappropriately scaled to the movement amplitude and velocity. 
Table Clinical details of patients $O N$ and OFF normal drug therapy

\begin{tabular}{|c|c|c|c|c|c|c|c|c|c|c|c|}
\hline \multirow[t]{2}{*}{ Patient } & \multirow[t]{2}{*}{ Sex } & \multirow{2}{*}{$\begin{array}{l}\text { Age } \\
(y r)\end{array}$} & \multirow{2}{*}{$\begin{array}{l}\text { Disease } \\
\text { duration (yr) }\end{array}$} & \multicolumn{4}{|c|}{ Off therapy } & \multicolumn{4}{|c|}{ On therapy } \\
\hline & & & & Rigidity & Bradykinesia & Tremor & $\begin{array}{l}\text { Hoehn } \\
\text { \& Yahr }\end{array}$ & Rigidity & Bradykinesia & Tremor & $\begin{array}{l}\text { Hoehn } \\
\text { \& Yahr }\end{array}$ \\
\hline $\begin{array}{l}\text { GL } \\
\text { VH } \\
\text { CM } \\
\text { AG } \\
\text { RC } \\
\text { MC } \\
\text { AR } \\
\text { JM } \\
\text { WS } \\
\text { WW }\end{array}$ & $\begin{array}{l}\mathbf{M} \\
\mathbf{F} \\
\mathbf{M} \\
\mathbf{M} \\
\mathbf{M} \\
\mathbf{M} \\
\mathbf{M} \\
\mathbf{M} \\
\mathbf{M} \\
\mathbf{M}\end{array}$ & $\begin{array}{l}42 \\
60 \\
67 \\
69 \\
54 \\
61 \\
53 \\
61 \\
56 \\
76\end{array}$ & $\begin{array}{r}10 \\
7 \\
9 \\
21 \\
18 \\
10 \\
5 \\
5 \\
3 \\
2\end{array}$ & $\begin{array}{l}3 \\
3 \\
3 \\
3 \\
3 \\
3 \\
2 \\
2 \\
1 \\
1\end{array}$ & $\begin{array}{l}3 \\
3 \\
3 \\
3 \\
3 \\
3 \\
3 \\
2 \\
2 \\
2\end{array}$ & $\begin{array}{l}1 \\
1 \\
1 \\
1 \\
1 \\
1 \\
0 \\
1 \\
0 \\
1\end{array}$ & $\begin{array}{l}\text { III } \\
\text { III } \\
\text { III } \\
\text { III } \\
\text { III } \\
\text { III } \\
\text { II } \\
\text { II } \\
\text { I } \\
\text { I }\end{array}$ & $\begin{array}{l}0 \\
0 \\
0 \\
0 \\
0 \\
0 \\
0 \\
0 \\
0 \\
0\end{array}$ & $\begin{array}{l}1 \\
1 \\
1 \\
1 \\
1 \\
1 \\
1 \\
0 \\
0 \\
0\end{array}$ & $\begin{array}{l}0 \\
0 \\
0 \\
0 \\
0 \\
0 \\
0 \\
0 \\
0 \\
0\end{array}$ & $\begin{array}{l}\text { I } \\
\text { I } \\
\text { I } \\
\text { I } \\
\text { I } \\
\text { I } \\
\text { I } \\
0 \\
0 \\
0\end{array}$ \\
\hline
\end{tabular}

Rigidity, tremor and bradykinesia were graded clinically on a scale from 0-3.

\section{Materials and methods}

Wrist flexion movements were studied in 10 patients with Parkinson's disease (mean age 60 years, range 42-76) who had a pronounced positive response to levodopa therapy. The clinical features of these patients are listed in the table. All were studied when bradykinetic 12-16 hrs after the last oral dose of levodopa (OFF therapy) and then when mobile $30 \mathrm{~min}-1 \mathrm{hr}$ after administration of the drug (ON therapy). The results were compared to a group of eight normal subjects (mean age 47 years, range 30-63) with no history of neurological disease. The mean age of this normal group was lower than that of the patients. This was because four of the normals were aged between 30 and 40 years whilst the other four were in their sixties. Since there was no difference between the performance of either subgroup on any task, their data were combined.

Subjects were seated in a chair with the forearm held semipronated and strapped to a platform which allowed only wrist flexion and extension movements. The fingers were encased in a rigid splint. Electromyographic activity was recorded with surface electrodes placed over the flexor agonists (flexor carpi radialis and flexor digitorum superficialis) and over the extensor antagonists (extensor carpi radialis and extensor digitorum communis). A coaxial potentiometer measured the angle of the wrist. Joint position, joint velocity (electronically derived from the joint position signal), and rectified EMG signals were recorded by a PDP 12 computer with a sampling rate of $500 \mathrm{~Hz}$ per channel. The signals were preamplified (Devices 3160 with a time constant of $60 \mathrm{~ms}$, low pass filtered to attenuate the signal by $3 \mathrm{~dB}$ at $2.5 \mathrm{kHz}$ ), then amplified (Devices 3120 amplifier) and processed (Devices signal processor type 4010).

Subjects were asked to flex the wrist as rapidly as possible in their own time through an angle of $15^{\circ}$ or $60^{\circ}$. The starting angle was $30^{\circ}$ extension, and a visual display of wrist position was available on an oscilloscope screen before them. Movements were made either with no load, or with a large opposing (extensor) torque of $2 \cdot 2 \mathrm{Nm}$ supplied by a torque motor (Printed Motors type G12M) mounted beneath the wrist joint. When the load was applied, a stop was placed behind the starting position so that subjects did not have to oppose the load until movement began. Subjects were allowed a few practice trials to familiarise themselves with the apparatus, then 15 single trials were collected under each condition.

Measurements were made on each single record using the computer display unit. Duration of the EMG bursts was measured by eye. The size of the bursts was measured by integrating the rectified EMG records. There was very little or no resting background activity in either flexor or extensor muscles in both patient and normal groups (see Results section). Student's $t$ test (paired or unpaired, as appropriate) was used to analyse the data.

\section{Results}

\section{(1) Normal subjects}

All normal subjects performed the wrist movements with a single biphasic or triphasic pattern of EMG activity (fig 1A). This began with a burst of activity in the agonist (flexor) (AG1) and was followed by activity in the antagonist (extensor) (ANT1). A small inconsistent second burst (AG2) sometimes was observed in the agonist. There was a tendency for our normal subjects to overshoot the target (by 6-7 $7^{\circ}$ in unloaded movements). As in previous studies, ${ }^{5}$ the peak velocity increased considerably in the $60^{\circ}$ movement compared with the $15^{\circ}$ movement whether or not load was applied (fig 2).

The first agonist burst was modulated under different conditions. Its duration and amplitude increased when large movements were made, or when the load was added (figs 3 and 4). The antagonist burst decreased in size or disappeared during the large movement made without any load (fig 1). When the opposing torque was applied, the antagonist activity became small or disappeared even with $15^{\circ}$ movements.

\section{(2) Parkinson's disease OFF therapy}

All the patients in this study had a recognisable burst pattern in the EMG from agonist and antagonist muscles (fig 1B). In eight patients rapid wrist movements, particularly to the $60^{\circ}$ target, were not smooth 

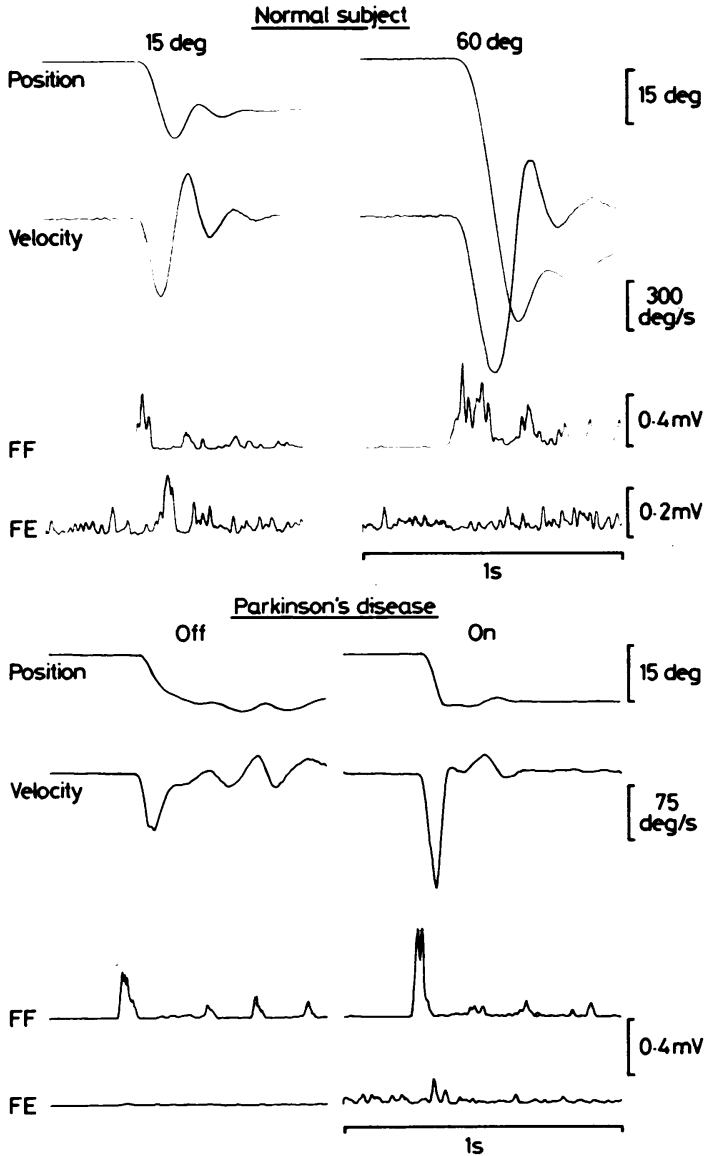

Fig 1 Single rapid wrist flexion movements in a normal subject $(A)$ and in a patient with Parkinson's disease when $O F F$ and $O N$ drug therapy (B). In A, a movement of $15^{\circ}$ (left) has been contrasted with a movement of $60^{\circ}$ (right). In $B, a 15^{\circ}$ movement made when OFF drugs (left) has been contrasted with the same movement made when $O N$ drugs. Records are, from top to bottom, wrist position, velocity and rectified surface EMG activity from the flexor and extensor muscles in the forearm. The EMG activities have been filtered digitally with 3-point moving average filter. Background activity can be seen in the extensor muscles before onset of movement. This is because subjects began to move from an initial angle of $30^{\circ}$ extension.

and the end point was reached in a stepwise manner (fig 1B). In two patients all movements were made smoothly and had a single triphasic EMG pattern. Only the first EMG burst and the resulting movement have been analysed. The background level of EMG activity in the antagonist muscle during the $200 \mathrm{~ms}$ period prior to onset of agonist activity was extremely small or absent. In those individuals in whom some activity was present, it was the same in the patients as in normals.
Rapid wrist movements were slower and slightly smaller in the patient groups than in the normals (fig 2). Despite the overall slowness of movement, the mean percentage increase in velocity when $60^{\circ}$ movements were compared with $15^{\circ}$ movements was the same in the patients as in the normals $(p>0.05)$. (Normal increase in velocity in unloaded movements was $272 \pm 21 \%$ (SE), in patients OFF medication it was $297 \pm 32 \%$, and in patients ON medication 245 $\pm 24 \%$. In loaded movements normals increased peak velocity by $192 \pm 28 \%$, patients OFF by $237 \pm$ $19 \%$, and patients $\mathrm{ON}$ by $216 \pm 16 \%$. Note that these figures are means calculated from the percentage increase in each subject. This is a slightly different value from the slope of the line in fig 2 . The raw data illustrated in this figure are calculated from the overall mean velocities at $15^{\circ}$ and $60^{\circ}$ in each group.)

Consistent with the changes in amplitude and velocity of movement, the first agonist burst of EMG in the Parkinsonian patients increased in duration and size both for larger movements and for movements against a load (figs 3 and 4). Detailed analysis of the results showed that for a given movement the duration of the first agonist burst was the same in the patients as in normal subjects in all conditions which were tested. The percentage increase in the amplitude of the first agonist burst for movements of different size or agonist load also was similar in patients and in normal subjects. In the absence of load, the EMG necessary for a $60^{\circ}$ movement was $175 \pm 26 \%$ (mean $\pm 1 \mathrm{SE}$ ) larger than that for a $15^{\circ}$ movement in the control group, and $189 \pm 46 \%$ larger in the patient group. When load was used, the additional EMG activity necessary to overcome the load was $105 \pm$ $19 \%$ for the control group and $377 \pm 175 \%$ for the patients in movements through $15^{\circ}$, and $50 \pm 13 \%$ and $134 \pm 58 \%$ in movements through $60^{\circ}$. (p > $>0.05$ for all comparisons between normals and patients.) (Note again that these increases are means calculated from percentage changes in each individual. They are slightly different from the raw data in fig 4 calculated from overall mean agonist burst sizes in each task.) Even though the movements in the Parkinsonian group were slower, the absolute size of the EMG bursts was similar to, rather than smaller than, that of the control group (fig 4).

\section{(3) Patients with Parkinson's disease ON therapy}

When patients were ON drug therapy a number of changes were seen. The changes were small and because of the spread of data between individual subjects, all comparisons were made on paired data from the same individual $O N$ and OFF medication. When ON there was a decrease in the number of EMG bursts in each movement and a small increase in 


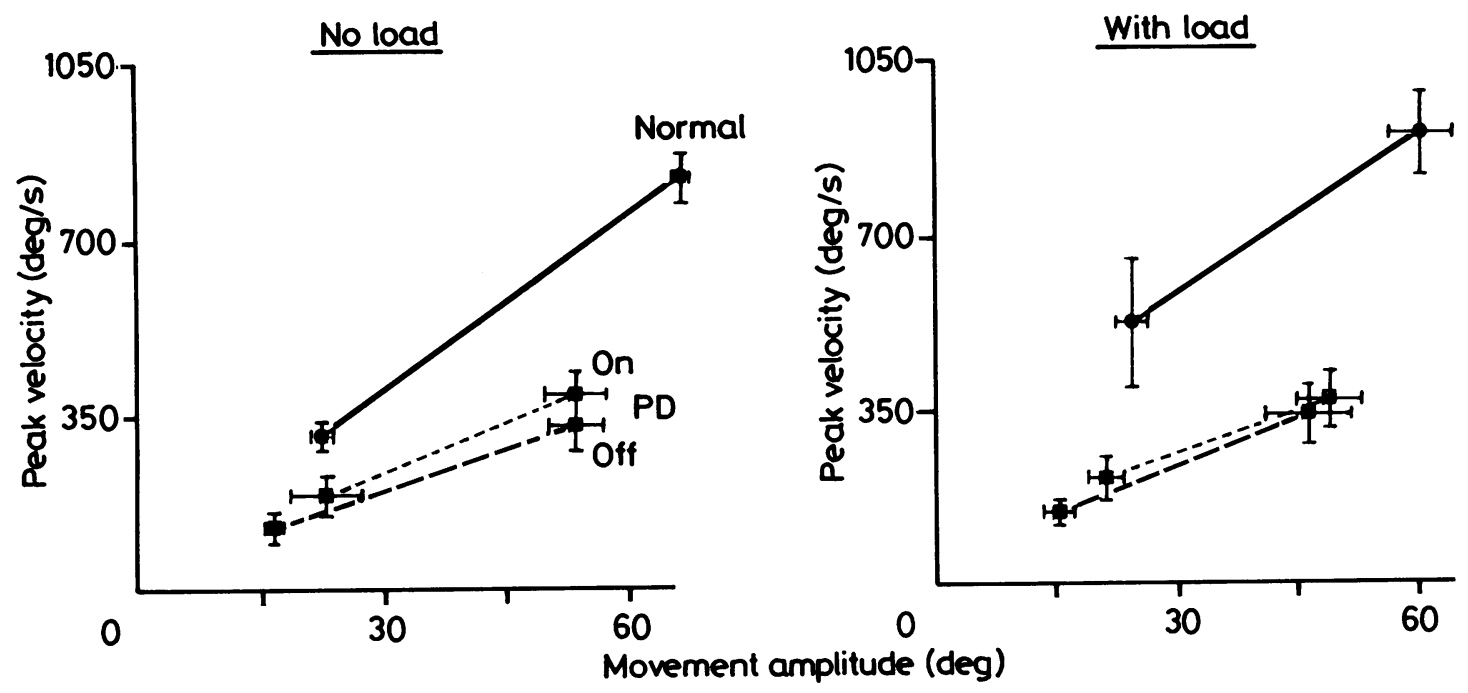

Fig 2 The relationship between movement amplitude (abcissa) and velocity (ordinate) for the $15^{\circ}$ and $60^{\circ}$ wrist flexion tasks

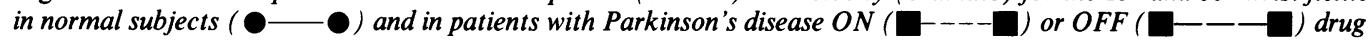
therapy. Movements with an opposing load of $2.2 \mathrm{Nm}$ are plotted on the right whilst unloaded movements are on the left. The points are mean $\pm 1 S E$ of the group averages. Straight lines have been used for clarity to join the points for the $15^{\circ}$ and $60^{\circ}$ task. The relationship between movement amplitude and velocity in such tasks may not, however, be completely linear.
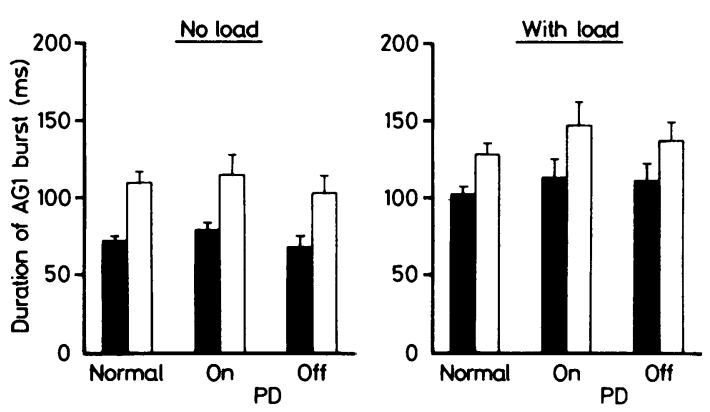

Fig 3 The duration of the first agonist EMG burst in normal subjects and in patients with Parkinson's disease $O N$ and OFF drug therapy. Data from unloaded movements is plotted on the left; data from movements made against an opposing torque of $2.2 \mathrm{Nm}$ are on the right. Each pair of bars represents the mean $\pm 1 S E$ of the group data from the $15^{\circ}$ (hatched columns) and $60^{\circ}$ (open columns) tasks. In each pair of bars, the duration of the burst in the $60^{\circ}$ task is longer than that in $15^{\circ}$ task (paired t test $p<0.05$ ). Similarly, the duration was longer in movements made with a load compared with the same movements without load (paired $t$ test $p<0.05$ ). Group comparisons between equivalent movements in patients and normals, or paired comparisons between patients $O N$ and $O F F$ therapy revealed no change in burst duration. ( $p>0.05)$. movement speed (average increase over all conditions

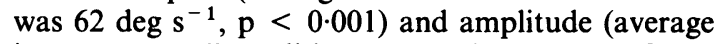
increase over all conditions was $5.6^{\circ}, \mathrm{p}<0.01$ ) (fig 2). The EMG bursts were larger (average increase over all conditions was 3.4 uV.s, p < 0.05) (fig 4), but their duration was unchanged (fig 3 ).

\section{Discussion}

It is well known that rapid intentional limb movements in patients with Parkinson's disease are slower than normal..$^{1-4}$ However, it is not clear why this is so. In their pioneering study Draper and Johns ${ }^{1}$ asked patients to perform rapid pronation/supination movements of the wrist through a range of target angles. In normal subjects, the peak velocity of the movement increased by a factor of four over the range studied. In patients, this increase was barely twofold. They concluded, "The patients' velocity was not only reduced, but their ability to modify velocity to meet changing demands was also seriously limited." In a later study, Flowers ${ }^{3}$ also concluded that "the rate of movement was not varied for (movements of) different amplitudes". The implication from this statement is that patients with Parkinson's disease can only execute movements of all amplitudes at a single slow speed. That is there appeared to be a 


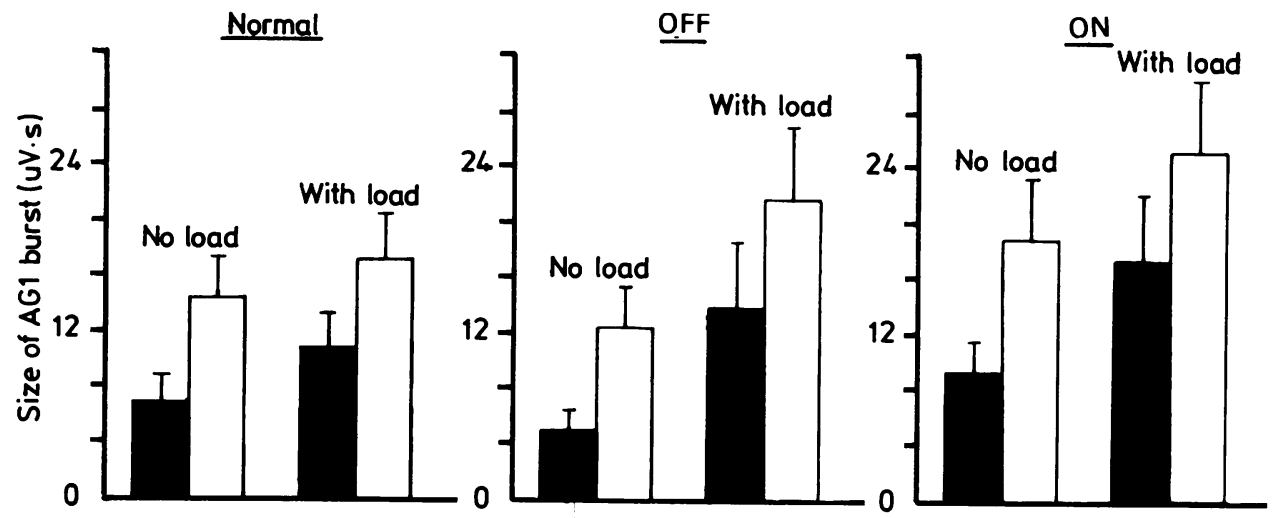

Fig 4 Average ( $\pm 1 S E$ ) absolute size of first agonist EMG burst, expressed as the area under the rectified surface EMG record, in normal subjects and in patients with Parkinson's disease $O N$ and $O F F$ therapy. Each pair of bars represents data from $15^{\circ}$ (hatched columns) and $60^{\circ}$ (open columns) movements in each condition. Paired comparisons reveal an increase in size of the burst in $60^{\circ}$ compared with $15^{\circ}$ movements in normal subjects and patients whether ON or OFF $(p<0.01)$. Similarly, there was an increase in burst size when movements were loaded $(p<0.05)$ in all groups. The average size of the agonist burst in the normal group was not different from that in the patients either $O N$ or OFF therapy $(p>0.05)$. Paired comparisons of agonist burst size in the $O N$ versus the OFF conditions are as follows: $15^{\circ}$ unloaded $p<0.05,60^{\circ}$ unloaded $p<0.01,15^{\circ}$ and $60^{\circ}$ loaded $p>0.05$.

saturation in the peak velocity of movement.

This conclusion has been taken up by other authors,${ }^{67}$ although careful reading of Flowers' original paper shows that it is not, in fact, true. The patients increased their mean peak velocity of movement by a factor of four over the range studied, as did the normal group (see fig 7 in ref 3). The findings in the present paper are in agreement with this. Comparing movements of $15^{\circ}$ and $60^{\circ}$, there was approximately a three fold increase in the peak movement velocity in both normal and patient groups. Hallett and Khoshbin ${ }^{2}$ also found that many patients with Parkinson's disease increased peak movement velocity with distance moved. A more appropriate conclusion, then, is that movement velocity of patients with Parkinson's disease is related to amplitude, but that this relationship is displaced from that seen in normal subjects.

In this paper we have related this finding to the underlying EMG activity in simple wrist flexion movements. In normal subjects the single triphasic pattern in the agonist and antagonist muscles responsible for fast arm movements about a single joint is not a fixed entity. The size and the timing of each burst can vary independently. ${ }^{7}$ The size of the first agonist burst increases ${ }^{8}$ while the size of the first antagonist decreases ${ }^{9}$ with the amplitude of the movements. The duration of the first agonist burst is constant when small movements of about $15^{\circ}$ and $30^{\circ}$ are performed, ${ }^{78}$ but changes when very large movements are made, ${ }^{510}$ or when movements are performed against a load and under conditions of muscle fatigue. ${ }^{5}$

Despite slowness of movement in all the tests performed, the patients with Parkinson's disease could change the amplitude and duration of the first agonist burst appropriate to the size of the movement and the background load, as in normal subjects. Thus patients with Parkinson's disease modulated EMG activity during single joint movements in the same way as normals. Even though the movements in the Parkinsonian group were slower, the absolute size of the EMG bursts was similar to, rather than smaller than, that of the control group (fig 4). Several possible reasons could explain this finding. First there could have been a difference in the skin resistance or in electrode placement which produced larger EMG signals in the patients with Parkinson's disease than in normals. Second, there may have been an increase in joint stiffness or resistance to stretch of the antagonist muscle in the patients, necessitating larger agonist activity to move the joint through the required angle. Changes in stiffness of antagonist muscles could not have been due to increased levels of background EMG activity in the patient group, since they were the same as normal. However, there may be changes in intrinsic muscle stiffness in patients with Parkinson's disease which could contribute to the phenomenon. ${ }^{11}$ 
Third, there may have been an abnormal force: EMG relation in the muscles of the patients, as has been reported for the paretic limbs of hemiparetic individuals. ${ }^{6}$ As we are concerned here with changes in the size of the EMG bursts rather than their absolute magnitude, this phenomenon will not be discussed further in this paper.

Since none of the patients showed any clinical evidence of muscle weakness in their arms, and they were all capable of producing large bursts of EMG activity, the question arises as to why they do not use these bursts to make rapid movements? Although minor perceptual changes have been documented in Parkinson's disease $\mathrm{e}^{12}$ the patients appeared to understand fully the nature of the task. They knew what to do and they realised when they failed. One possibility is that the correct signal was sent out from the motor cortex but that it failed to activate the spinal cord correctly because of some defect in the corticospinal pathway. However, we have shown previously ${ }^{13}$ that in Parkinson's disease the cortico-motoneuron connection is intact even in bradykinetic patients who were OFF treatment. Similarly, there is little defect in intrinsic spinal cord mechanisms, as seen in the recruitment order of the motoneurons, ${ }^{14}$ which would explain the slowness of movements in Parkinson's disease.

It is probable that it is the command sent to the motor cortex which is wrong in Parkinson's disease. The patients appear to underestimate what muscle activity is required for a particular movement. There is a breakdown of the link between perceptual appreciation of what is needed and the delivery of appropriate instructions to the motor cortex. Why this is so is not clear. Single unit recordings for the output zones of the basal ganglia (the globus pallidus and substantia nigra reticulata) have not been shown to correlate closely with levels of EMG activity. ${ }^{15}$ Perhaps the premotor cortex and supplementary motor area, deprived of their normal basal ganglia input by Parkinson's disease, do not compute the command signal to the motor cortex to match the size of the movement required.

Another finding deserves discussion. Evarts et al, ${ }^{16}$ who made a detailed study of reaction times in a wrist pronation/supination movement, found that reaction times in Parkinson's disease were not a useful indicator of therapeutic efficiency, and suggested that movement times might provide a more useful objective measure. Baroni et $\mathbf{l l}^{17}$ studied the effects of levodopa on rapid arm abduction movement and found that movement times decreased when patients were ON therapy. In the present experiments we also found that the peak velocity of movement increased (hence movement time decreased) when patients were ON therapy, but the amount of change was relatively small compared with the OFF condition. This contrasted with the much more dramatic change in the patients' overall clinical state, as recorded in the Hoehn \& Yahr gradings of the table. We would conclude that the changes in the velocity of a single joint movement may not provide a good description of overall clinical change in Parkinson's disease. There must be some other abnormalities in motor control to explain the great disability of movement when patients are OFF drug therapy.

We thank particularly all the patients who participated so willingly in these experiments. The specialised equipment was designed by Mr HC Bertoya and built by $\mathrm{Mr} \mathrm{R}$ Miller. The work was supported by the Medical Research Council and the research funds of the Bethlem Royal and Maudsley Hospitals. JCR is a Royal Society University Research Fellow.

\section{References}

${ }^{1}$ Draper IT, Johns RS. The disorders of movement in Parkinsonism and the effect of drug treatment. Bull Johns Hopk Hosp 1964;115:465-80.

${ }^{2}$ Hallett M, Khoshbin S. A physiological mechanism of bradykinesia. Brain 1980;103:301-14.

3 Flowers KA. Visual "closed loop" and "open loop" characteristics of voluntary movement in patients with Parkinsonism and intention tremor. Brain 1976;99: 269-310.

${ }^{4}$ Berardelli A, Rothwell JC, Day BL, Marsden CD. Movements not involved in posture are abnormal in Parkinson's disease. Neurosci Lett 1984;47:47-50.

${ }^{5}$ Berardelli A, Rothwell JC, Day BL, Kachi T, Marsden CD. Duration of the first agonist EMG burst in ballistic arm movements. Brain Res 1984;304:183-7.

${ }^{6}$ Tang A, Rymer WZ. Abnormal force-EMG relations in paretic limbs of hemiparetic subjects. J Neurol Neurosurg Psychiatry 1981;44:690-8.

${ }^{7}$ Brown SH, Cooke JD. Amplitude and instructiondependent modulation of movement-related EMG activity in humans. J Physiol (Lond) 1981;316:97-107.

${ }^{8}$ Hallett M, Marsden CD. Ballistic flexion movements of the human thumb. $J$ Physiol (Lond) 1979;294:33-50.

${ }^{9}$ Marsden CD, Obeso JA, Rothwell JC. The function of the antagonist muscle during fast limb movements in man. J Physiol (Lond) 1983;335:1-13.

${ }^{10}$ Brown SH, Cooke JD. Initial agonist burst duration depends on movement amplitude. Exp Br Res 1984; 55:523-7.

${ }^{11}$ Watts RL, Wiegner AW, Young RR. Changes in passive muscle properties associated with rigidity of Parkinson's disease. VIII Int. Symposium on Parkinson's Disease 1985:57.

${ }^{12}$ Marsden CD. Function of the basal ganglia as revealed by cognitive and motor disorders in Parkinson's disease. Can J Neurol Sci 1984;11:129-35.

${ }^{13}$ Dick JPR, Cowan JMA, Day BL, Kachi T, Rothwell JC, 
Marsden CD. The corticomotoneurone connection is normal in Parkinson's disease. Nature 1984;310:407-9.

${ }^{14}$ Petajan JH. Motor Unit Control in movement disorders. In: Desmedt JE, ed. Motor Control Mechanisms in Health and Disease, New York: Raven Press, 1983:897-905.

${ }^{15}$ DeLong MR, Georgopoulos AP, Crutcher MD, Mitchell SJ, Richardson RT, Alexander GE. Functional organisation of the basal ganglia: contributions of single-cell recording studies. In: Functions of the basal Ganglia, Ciba Symposium, 107. London: Pitman, 1984:64-82.

${ }^{16}$ Evarts EV, Teravainen H, Calne DB. Reaction time in Parkinson's disease. Brain 1981;104:167-86.

${ }^{17}$ Baroni A, Benvenuti F, Fantini L, Pantaleo T, Urbani F. Human ballistic arm abduction movements: effects of L-Dopa treatment in Parkinson's disease. Neurology (Cleveland) 1984;34:868-76. 